\title{
is Research Square \\ On the Regularity of the Electron Configuration of Atoms in the Periodic Table
}

\section{Toshihiro Konishi ( $\nabla$ ywkjast@hotmail.co.jp )}

Konishi Technical Consultant

\section{Ryosuke Miura}

Teikyo University

\section{Research Article}

Keywords: transition elements, current periodic table, three dimensional structure

Posted Date: June 17th, 2021

DOl: https://doi.org/10.21203/rs.3.rs-595410/v1

License: (9) This work is licensed under a Creative Commons Attribution 4.0 International License. Read Full License 
Title

On the regularity of the electron configuration of atoms in the periodic table

\author{
Author's affiliations \\ Toshihiro Konishi \\ Konishi Technical Consultant \\ Sole proprietor \\ 2-18-4 Kouraku, Bunkyo-ku, Tokyo Japan 112-0004 \\ ywkjast@hotmail.co.jp \\ Ryosuke Miura \\ Faculty of Pharmacy, Teikyo University \\ Student \\ 2-11-1 Kaga, Itabashi-ku, Tokyo Japan 173-8605 \\ ywkjast924@gmail.com
}

\begin{abstract}
The current periodic table does not necessarily have a clear position for transition elements.

Therefore, the purpose of this paper is to use the basic principle discovered by Mendeleev as it is and to create a periodic table with consistency for transition elements.
\end{abstract}

By setting some hypotheses, it was found that transition elements also have regular periodicity, so we succeeded in clarifying the energy level of electrons in each orbit.

In addition, by utilizing its periodicity, the electron configuration for each orbit was predicted for unknown elements.

In this paper, we did not take the conventional idea of electron orbitals, that is, the idea of forming a hybrid orbital, but assumed a new orbital.

Since the state in which electrons fit in orbits and stabilize is defined as an octet, this idea was used as the basic principle in this paper, but the hypothesis that "there are only three orbits in each shell" was established and verified.

The calculation of the energy level of the electrons on the orbit became extremely easy, and the order of each orbit could be clarified.

It was also found that the three-dimensional structure of the molecule may be visualized by paying attention to the valence electrons of the outermost shell of the element and the octet of the stability condition.

Therefore, in this paper, by slightly expanding the structural formula of Kekulé, it 
became possible to easily determine whether or not the molecule synthesized by the bond between elements is stable.

In addition, it has become possible to predict the three-dimensional structure of the molecule as well.

Furthermore, not only will it be easier for students studying chemistry to understand complex chemical reactions, but it will also be useful for researchers in the development and research of new drugs.

\section{Introduction}

The periodic table currently in use is created based on the number of electrons existing in the orbit of the outermost shell.

It is assumed that the electron shell is a collection of atomic orbitals ( $\mathrm{s}$ orbital, $\mathrm{p}$ orbital, $d$ orbital, f orbital ...). In addition, a method has been adopted to explain the chemical reaction formula on the premise of the electron orbital model.

However, the method was not only too complicated for researchers, but there were many cases of inconsistency. In such a case, we treated it as an exception as a special case and did not pursue it deeply.

The transition elements in the periodic table have also been treated as special cases without discussing the clear regularity of their periodicity.

This paper reviews the conventional structural principles a little and reconstructs the structures of atoms and molecules to examine the phenomena that have been treated as exceptions. By demonstrating that the events treated as exceptions are events with regular periodicity, the periodic table becomes even easier to use, and the hypothesis of this paper becomes meaningful.

Many periodic tables have already been published, but they all have in common that the number of electrons in the outermost shell has a large effect on the properties of the element and that the number is limited.

That is, the number of electrons existing in the orbit of the outermost shell is limited to 8 (however, only the $\mathrm{K}$ shell is 2 ), and the fully filled state is called an octet or a closed shell. This term is also used in this paper.

However, the handling of transition elements, lanthanoids, and actinides is extremely complicated, and it is inconvenient to use them as a list.

According to this structural principle, a mysterious phenomenon occurs in the orbit of the electron shell. In other words, even though the orbit still has room to store electrons, it enters another orbit for no particular reason. 
Furthermore, it is difficult to understand that a complex orbital called a hybrid orbital is formed.

For example, carbon elements form hybrid orbitals called $\mathrm{sp}$ orbitals, $\mathrm{sp}^{2}$ orbitals, and $\mathrm{sp}^{3}$ orbitals, and discuss elemental bonds by creating completely new orbitals and changing their shapes

In this paper, in order to remove these complications and create a more consistent periodic table, we set simple conditions and verified its usefulness.

Just as a plane is defined in two dimensions, the common sense idea that space is defined in three dimensions applies to the existence of orbits.

At the same time, the easy-to-understand concept of an octet that maintains a stable state is the basis of the hypothesis of this paper

In addition, in order to visualize how electrons revolve on the same orbit while rotating in opposite directions, the orbit when the electrons revolve in the same direction as the rotation direction is named the first small orbit. The orbit that revolves while rotating in the opposite direction is named the second small orbit.

This paper illustrates the bonds between atoms and visualizes the structure of the constituent molecules to verify them using a method that compares them with the structures of molecules that have already been found.

By diagramming the bonds between atoms and visualizing the structure of the constituent molecules, we will verify using a method that compares with the already known molecular structure.

The hypothetical conditions and research policy are as follows.

1: The number of electrons existing in the orbit is 2 in the $\mathrm{K}$ shell and up to 8 in the orbits of the other shells, and the state where the orbit is filled with electrons is called an octet.

2: Each shell has orbits around 3 axes and there is no more. That is, there are only three orbits. Since the orbit of the K shell is in an octet state with two electrons, it can store up to 6 electrons, but the other shells can store up to 24 electrons.

3: Electrons move in orbit as standing waves.

4: Electrons are buried from orbits with low energy levels.

5: When the orbit is filled with electrons and becomes an octet, a barrier is formed in the shell. Energy is required for electrons to break through the barrier. Therefore, the energy level may be higher in the inner shell orbit than in the outer shell orbit.

6: When an electron is stored in the orbit of the inner shell, when it becomes an octet, it enters the orbit of the next outer shell, that is, only one orbit can enter the same shell. 
The first to sixth terms are the conditions for finding the energy level.

The following items are the conditions added to visually verify whether the periodic table created under the above conditions is appropriate.

The orbit consists of a first small orbit and a second small orbit depending on the direction of electron rotation. ..

The first small orbit in which the electron fits is named the first small orbit. The remaining small orbit is named the second small orbit. That is, each small orbit can store up to four electrons. And this state is called a quartet.

Octet is defined as "there are eight electrons that rotate in opposite directions on the same orbit", but if nothing is done, electrons that rotate clockwise and counterclockwise will coexist on the same orbit. It is inconvenient when considering the bonds between atoms. Therefore, only those whose electrons rotate in the same direction are taken out on the small orbit.

In addition, the orbital planes of the first and second small orbits shall sandwich the atomic nucleus.

7: Electrons occupy the first and second small orbits alternately.

8: The electron revolves while revolving on each small orbit.

9: The direction of rotation of electrons on the small orbit may be the same as or opposite to the direction of revolution.

10: There are clockwise and counterclockwise directions of electron revolution in the first small orbit.

11: It stabilizes when the orbit is filled with electrons and becomes an octet. Since the shell forms a barrier, electrons are stored in the outermost shell. Therefore, the energy required to destroy the barrier is required for the electrons to fit in the orbit of the shell. If the electron does not have enough energy to destroy the barrier, it will fall into the orbit of the outermost shell, which has a lower energy level.

Items 1 to 6 follow Miura's hypothesis, and subsequent items are added to test the hypothesis.

Main paper

1: Background

In the atomic model in quantum mechanics, the periodic table is created based on the principal quantum number, azimuth quantum number, magnetic quantum number, and so on. Each shell has an electron orbital, which is named s orbital, $p$ orbital, $d$ orbital, $f$ orbital, etc., but in this paper, the orbital defined by the hypothesis is used instead of this electron orbital. 
In the conventional method of calculating the number of orbitals, the total number of orbitals is $n^{2}$ where $n$ is the number of shells. This means that there are too many orbitals, and even though there is room to store electrons, there are orbitals that remain vacant. The conventional idea that electrons do not enter the $f$ orbit when the outermost shell becomes $\mathrm{P}$ shell or more seems a little unnatural. That is, the orbit itself may not exist.

Taking xenon as an example, xenon belongs to the $\mathrm{O}$ shell, so $\mathrm{n}=5$.

The total number of electrons that can be stored in orbit for xenon atomic number 54 is 110 .

The number of electrons that can be stored only in the outermost shell is $2 n^{2}$, that is, 50, and 110 electrons can be stored in total.

Electrons are not stored in the forbital of the inner shell $\mathrm{N}$ shell, but are stored in the s orbital and $\mathrm{p}$ orbital of the outermost $\mathrm{O}$ shell.

The number of electrons that can be stored in the outermost shell is defined as $2 n^{2}$, and a periodic table is created.

However, the reality is that up to eight electrons are stored in the outermost shell of all elements.

From this, we will review and verify the definition of the number of orbitals and the number of electrons that can be stored in those orbitals.

Only the arrangement of electrons in the outermost shell is taken up and whether it is an octet or not, but little attention is paid to the arrangement of electrons housed in the orbit of the inner shell.

Rather, the word octet has no meaning in the inner shell.

However, it is more natural to think that the inner shell is also stable while maintaining the octet state.

The first question of the conventional periodic table is that electrons do not enter the $\mathrm{f}$ orbit above the $\mathrm{P}$ shell. That is, the orbit of the inner shell is not used. This may mean that the orbit itself does not exist.

The second question is that the role of the octet in the placement of electrons in the inner shell is unclear.

In order to clarify and resolve these questions, the following two hypotheses have been established in this paper.

To answer the first question, "There are only three orbitals in each shell that are independent of each other, that is, there are only three orbitals around the $\mathrm{X}, \mathrm{Y}$, and $\mathrm{Z}$ axes for each shell. We limited the number of orbits and eliminated the existence of unnecessary orbits. 
The second question is, "When the orbit of the inner shell becomes an octet, the inner shell forms a barrier", and when the orbit of the inner shell becomes an octet, electrons cannot enter another orbit of the inner shell.

The electron energy is insufficient to enter the inner shell where the barrier is formed. However, it is stored in the outer shell orbit because it is sufficient to enter the outer shell orbit. The opposite is also true due to the energy level relationship.

In this paper as well, the names of orbits are used using symbols such as K, L, M, and $\mathrm{N}$ corresponding to the shell.

However, the orbits are identified by adding the subscripts ${ }_{\mathrm{X}}, \mathrm{Y}$, and $\mathrm{Z}$ to the names of their shells.

That is, by using the display of $\mathrm{K}_{\mathrm{X}}, \mathrm{K}_{\mathrm{Y}}$, and $\mathrm{K}_{\mathrm{Z}}$, the orbit around the $\mathrm{X}$ axis of the $\mathrm{K}$ shell is represented by $\mathrm{K}_{\mathrm{X}}$.

We will verify whether the periodic table created based on the above hypothesis has periodicity for all elements in the order of atomic numbers.

However, even if we succeed in clarifying the order of the energy levels of electrons, it is almost impossible to accurately obtain the value itself with the current computing power.

Therefore, by using the electron configuration of the elements derived in this paper to schematize and visualize the molecules generated by the bonds between atoms, the obtained structural diagram and the structure and photographs of the actually obtained molecules can be obtained. Compare and verify consistency.

\section{2: Verification result}

\section{(1) Extended Periodic Table (See Table 1)}

When the periodic table was verified under the conditions defined in this paper, the same results as the conventional periodic table were obtained up to the elements of the third period.

The electrons fit neatly in the orbits of the $\mathrm{K}$ shell, L shell, and $\mathrm{M}$ shell, and the valence electrons of the outermost shell are the same.

For the electron of element lithium (Li) with atomic number 3, the $\mathrm{K}_{\mathrm{X}}$ orbit of the innermost shell of the first period becomes an octet, and the $\mathrm{K}$ shell that becomes helium (He) forms a barrier, so the third electron becomes a $\mathrm{K}_{\mathrm{Y}}$ orbit. Cannot enter and fits in the $\mathrm{L}_{\mathrm{X}}$ orbit of the outermost $\mathrm{L}$ shell.

Similarly, when the $\mathrm{L}_{\mathrm{X}}$ orbit becomes an octet, the $\mathrm{L}$ shell also forms a barrier.

Therefore, the electron of sodium $(\mathrm{Na})$ with atomic number 11 cannot enter the $\mathrm{K}_{\mathrm{X}}$ orbit of the $\mathrm{K}$ shell and the $\mathrm{L}_{\mathrm{X}}$ orbit of the $\mathrm{L}$ shell, which are the inner shells, and 
therefore fall into the $\mathrm{M}_{\mathrm{X}}$ orbit of the $\mathrm{M}$ shell.

Considering the energy level in this case, $\mathrm{K}_{\mathrm{X}}<\mathrm{L}_{\mathrm{X}}<\mathrm{M}_{\mathrm{X}}$.

The electrons of group 1 potassium $(\mathrm{K})$ and group 2 calcium $(\mathrm{Ca})$ of the elements of the 4th period fit in the $\mathrm{N}_{\mathrm{X}}$ orbit.

For transition elements after atomic number 21, the energy level becomes insufficient to fit in the $\mathrm{N}_{\mathrm{X}}$ orbit of the $\mathrm{N}$ shell as the number of electrons increases.

However, since it has enough energy to break through the barrier of the $\mathrm{K}$ shell inside it, the electron moves to the $\mathrm{K}_{\mathrm{Y}}$ orbit of the inner shell with a lower energy level and becomes an octet with two.

When the $\mathrm{K}_{\mathrm{Y}}$ orbit becomes a closed shell (octet) as the number of electrons increases, the $\mathrm{K}$ shell forms a new barrier, so the electrons of the next element scandium (Sc) cannot enter the $\mathrm{K}$ shell orbit.

This electron has enough energy to break through the barrier of the $\mathrm{L}$ shell, so it can enter the $\mathrm{L}_{Y}$ orbit, but there is not enough energy to fit in the $\mathrm{N}$ shell.

As the atomic number increases, the increasing electrons gradually fit into the $\mathrm{L}_{Y}$ orbit, and when it reaches zinc ( $\mathrm{Zn}$ ) that fills eight, the $\mathrm{L}_{Y}$ orbit becomes an octet, stabilizes, and the $\mathrm{L}$ shell forms a barrier again.

When the transition elements are settled in their respective orbits $\mathrm{K}_{Y}$ and $\mathrm{L}_{Y}$ and become stable, the electrons of the elements after gallium $(\mathrm{Ga})$ of group 13 in the 4th period move to the $\mathrm{N}_{\mathrm{X}}$ orbit of $\mathrm{N}$ shell again and are stored.

However, although the electron energy is not high enough to break through that barrier, it is sufficient to fit into the $\mathrm{N}_{\mathrm{X}}$ orbit of the outermost $\mathrm{N}$ shell.

The energy level at this time is $\mathrm{N}_{\mathrm{X}}(1 \& 2)<\mathrm{K}_{\mathrm{Y}}<\mathrm{L}_{\mathrm{Y}}<\mathrm{N}_{\mathrm{X}}(3 \sim 8)$.

The transition elements of the 5 th period also behave in exactly the same way as the 4 th period described above.

That is, after the electrons of groups 1 and 2 are contained in $\mathrm{O}_{\mathrm{X}}$ orbit, the transition elements move to the $\mathrm{K}_{\mathrm{Z}}$ orbit and become stable with two, then move to the $\mathrm{L}_{\mathrm{Z}}$ orbit and fill eight and stabilize.

After the transition elements are placed in their respective orbits, the group 13 element indium (In) is placed in the $\mathrm{O}_{\mathrm{X}}$ orbit again.

The energy level at this time is $\mathrm{Q}_{\mathrm{X}}(1 \& 2)<\mathrm{K}_{\mathrm{Z}}<\mathrm{L}_{\mathrm{Z}}<\mathrm{O}_{\mathrm{X}}(3 \sim 8)$.

For the 6th period, after the electrons of groups 1 and 2 are contained in the $\mathrm{P}_{\mathrm{X}}$, they move to the $\mathrm{M}_{\mathrm{Y}}$ orbit and become stable with 8 electrons.

At this point, the orbits of the $\mathrm{K}$ and $\mathrm{L}$ shells are all filled with electrons, so there are no orbits for storing electrons in both shells.

Therefore, the electrons of the elements after the transition element lanthanum (La) 
are stored in the $\mathrm{M}_{\mathrm{Y}}$ orbit and then move to the $\mathrm{N}_{\mathrm{Y}}$ orbit.

Here, too, a barrier is formed in the $\mathrm{M}$ shell when it reaches the gadolinium (Gd) in which eight electrons are stored.

The next element, terbium $(\mathrm{Tb})$, has one more electron than cadmium.

The electron moves to the $\mathrm{N}_{\mathrm{Y}}$ orbit of the outer shell.

When it reaches hafnium (Hf), which contains eight electrons in this $\mathrm{N}_{\mathrm{Y}}$ orbit, a barrier is formed in the $\mathrm{N}$ shell, so in the next element tantalum (Ta), the increased single electron moves to the $\mathrm{O}_{\mathrm{Y}}$ orbit of the outer shell.

When this $\mathrm{O}_{\mathrm{Y}}$ orbital is filled with eight electrons and becomes an octet, a barrier is formed in the $\mathrm{O}$ shell, so the next element thallium (Tl) moves to the outermost $\mathrm{P}_{\mathrm{X}}$ orbital again.

The energy level at this time is $\mathrm{P}_{\mathrm{X}}(1 \& 2)<\mathrm{M}_{\mathrm{Y}}<\mathrm{N}_{\mathrm{Y}}<\mathrm{O}_{\mathrm{Y}}<\mathrm{P}_{\mathrm{X}}(3 \sim 8)$.

In the 7 th period, the electrons behave in the same way as in the 6 th period.

That is, after the electrons of groups 1 and 2 are contained in the $\mathrm{Q}_{\mathrm{x}}$, they move to the $\mathrm{M}_{\mathrm{Z}}$ orbit, become stable at eight, and then move to the $\mathrm{N}_{\mathrm{Z}}$ orbit.

Again, when eight octets are used, they move to the $\mathrm{O}_{Z}$ orbit. When the number of electrons becomes stable with eight, the element nihonium $(\mathrm{Nh})$ of group 13 moves to the outermost $\mathrm{Q}_{\mathrm{X}}$ orbit again.

At this point, the electron orbitals of the $\mathrm{M}$ shell, $\mathrm{N}$ shell, and $\mathrm{O}$ shell are filled with all the electrons, and all the elements are stored in the orbits in the order of atomic numbers.

The energy level at this time is $\mathrm{Q}_{\mathrm{X}}(1 \& 2)<\mathrm{M}_{\mathrm{Z}}<\mathrm{N}_{\mathrm{Z}}<\mathrm{O}_{\mathrm{Z}}<\mathrm{Q}_{\mathrm{X}}(3 \sim 8)$.

It has now been found that the electron configurations of all identified elements and all atoms up to the 118th element Onegason $(\mathrm{Og})$ are orderly and consistent. This made it possible to predict the electron configuration of unknown elements.

Since the electron configuration of atoms after the 119th atomic number can be easily estimated according to the rules defined in this paper, an extended periodic table up to the atomic number 222 was created.

(2) Orbital notation and combination of elements

The periodic table created based on the assumptions described in the introduction not only clearly shows the periodicity of transition elements, but also makes full use of the idea of octets.

The periodic table currently in use also began with observing events and discovering their periodicity, and many scholars are still publishing extended periodic tables. But what we can say in common is that the table is complicated. Some even published 
periodic tables that are not arranged in atomic number order.

The periodic table created based on Miura's hypothesis is too orderly and easy to understand, so I thought that there might be a method that can be verified without directly finding the energy level with the help of a supercomputer. , We decided to compare the structure of the molecule inferred from the electron configuration of the atom defined in this paper with the structure of the actual molecule and verify its consistency.

In the extended periodic table of this paper, the electron configuration of atoms is orderly. From this, it can be easily imagined that the chemical reaction has a structure that is easy to understand graphically.

In this paper, the molecular structure is represented graphically to make it easier to visualize and understand. As a method for that, we will introduce a new notation table and verify the validity of the theory.

Therefore, consider a pair of small orbitals that sandwich the nucleus of an element.

In addition, each small orbit can store 4 electrons, and when the two small orbits in this pair are combined, it has 8 electrons, so it is considered to be equivalent to the orbit defined by Miura's hypothesis.

Therefore, the above-mentioned extended periodic table can be used as it is.

That is, if the orbit is in an octet state, four electrons will be stored in the first small orbit and four electrons will be stored in the second small orbit across the nucleus.

In other words, an octet is a state in which four electrons are stored in each of a pair of small orbits.

When elements combine to form a molecule, only the outermost valence electrons are involved.

Since the orbit of the outermost shell of an element does not lose its generality even if it is on the horizontal plane, the orbit of the outermost shell thereafter is described as being on the horizontal plane.

In this paper, it is possible to visualize the bonding state between elements by treating the small orbits of this pair as one unit and diagramming them.

It was found that the structural formula of the molecule can be expressed by defining a simple symbol.

Traditionally, [-] has been used as a symbol for covalent bonds, so this paper also uses the same symbol.

However, when bonding elements, a new symbol is defined to clarify the side that provides and the side that receives electrons.

That is, the symbols $[\rightarrow]$ and $[\leftarrow]$ indicate the direction given by the electron. 
A concrete example is shown using an oxygen element $(\mathrm{O})$ and an oxygen molecule $\left(\mathrm{O}_{2}\right)$.

Since the valence electrons of the oxygen element are hexavalent, they have 6 electrons in the outermost shell.

The electron configuration of the oxygen atom is shown in Fig. 1 by this notation.

In this paper, we defined that there are 3 electrons in the 1st small orbit and 3 electrons in the 2nd small orbit, so it can be represented graphically as follows.

The orbitals are shown as straight lines instead of circles to make it easier to understand the bonding state of atoms.

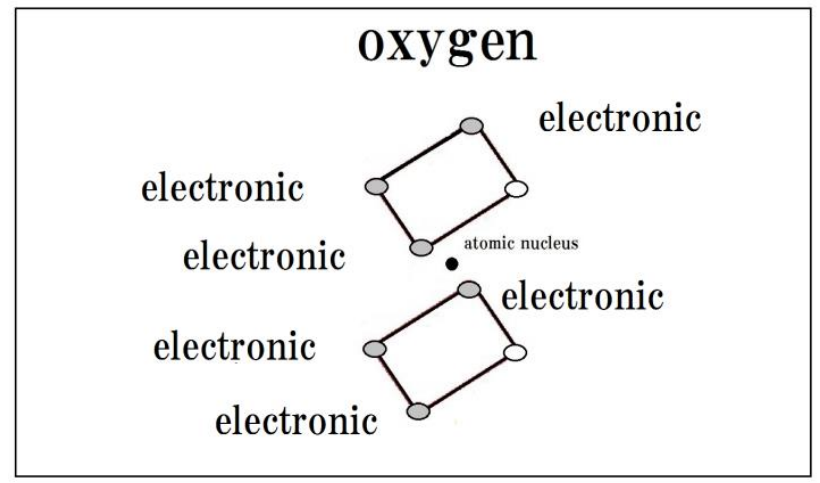

Fig. 1 oxygen atom

Oxygen molecules are formed by combining two oxygen elements.

Figure 2 shows a state in which oxygen electrons enter a small orbital vacant place and the orbital becomes stable, that is, the orbital becomes octet.

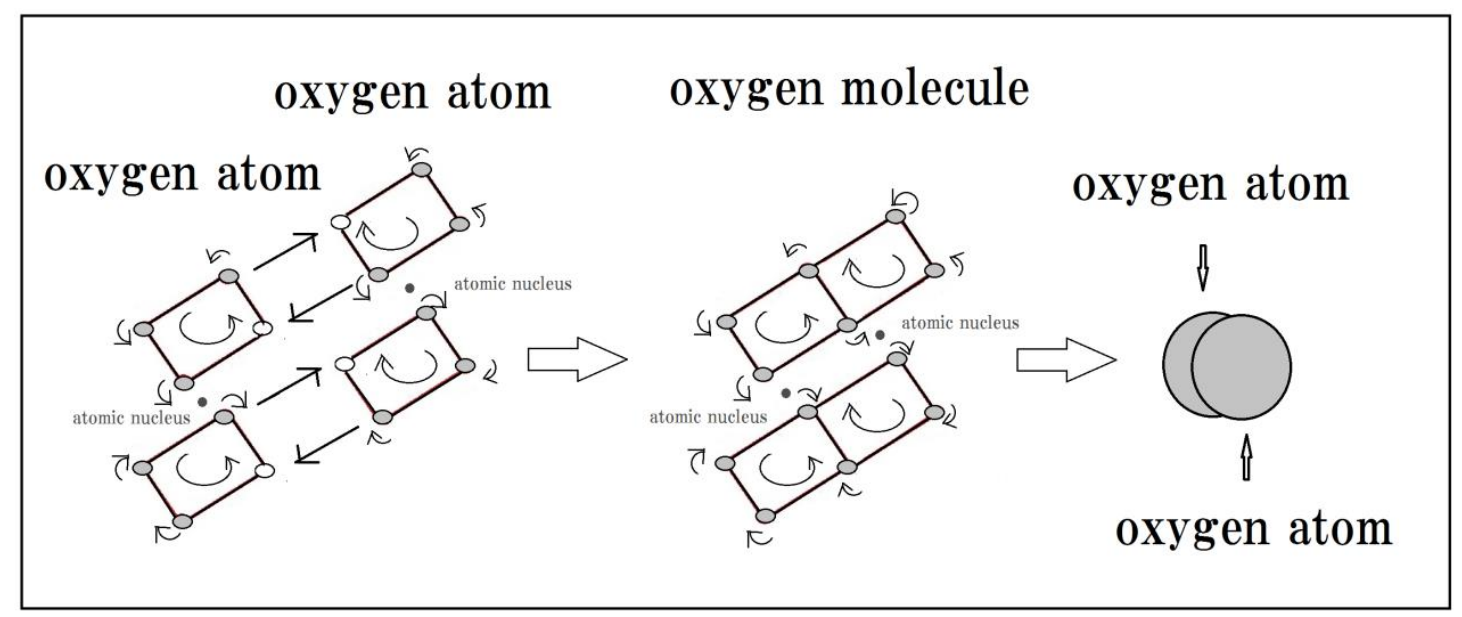

Fig. 2 oxygen molecules

The figure on the left side of Fig. 2 shows how two oxygen atoms combine to form an oxygen molecule.

The revolution direction of the electrons in the first orbit rotates in the same direction 
on the second orbit, but the rotation directions of the electrons are opposite to each other. Each direction of rotation is indicated by a large arrow and a small arrow.

One electron in the first small orbit enters the vacant place in the first small orbit on the right side, and one electron in the first small orbit on the right side enters the vacant place in the first small orbit on the left side. The orbitals shared by the oxygen molecules are in the same direction, and the rotation directions of the electrons on the orbitals are the same.

In the bond between elements, the direction of electron revolution must be the same. This satisfies the precondition that the same element has clockwise and counterclockwise rotation.

Therefore, each oxygen atom complements the deficient electrons with each other, and the first and second orbitals become quartets and become stable.

Both bonded small orbitals are filled with all electrons.

When the structural formula is expressed by the notation defined in this paper, the electrons in the first small orbit are $\mathrm{O} \rightarrow \mathrm{O}$ and $\mathrm{O} \leftarrow \mathrm{O}$, and since the electrons exchange with each other, they are covalent bonds. In this case, since it means a covalent bond, the same notation as the Kekule structural formula is used. That is, the upper side (first small orbit) can be written as $\mathrm{O}-\mathrm{O}$.

The lower electron undergoes exactly the same phenomenon as the upper electron and the lower side (second small orbit) can be written as $\mathrm{O}-\mathrm{O}$.

Therefore, the structural formula in this paper is $\mathrm{O}=\mathrm{O}$.

The above figure also suggests the three-dimensional structure of oxygen molecules. That is, two oxygen atoms are bonded side by side in parallel.

Furthermore, the case of methane $\left(\mathrm{CH}_{4}\right)$ will be verified.

The valence electrons of the carbon element are tetravalent, and have four electrons in the outermost shell.

In this paper, there are two electrons in the first orbit and two electrons in the second orbit.

Hydrogen electrons enter the vacant place of this small orbit, and the orbit becomes an octet and stabilizes. 


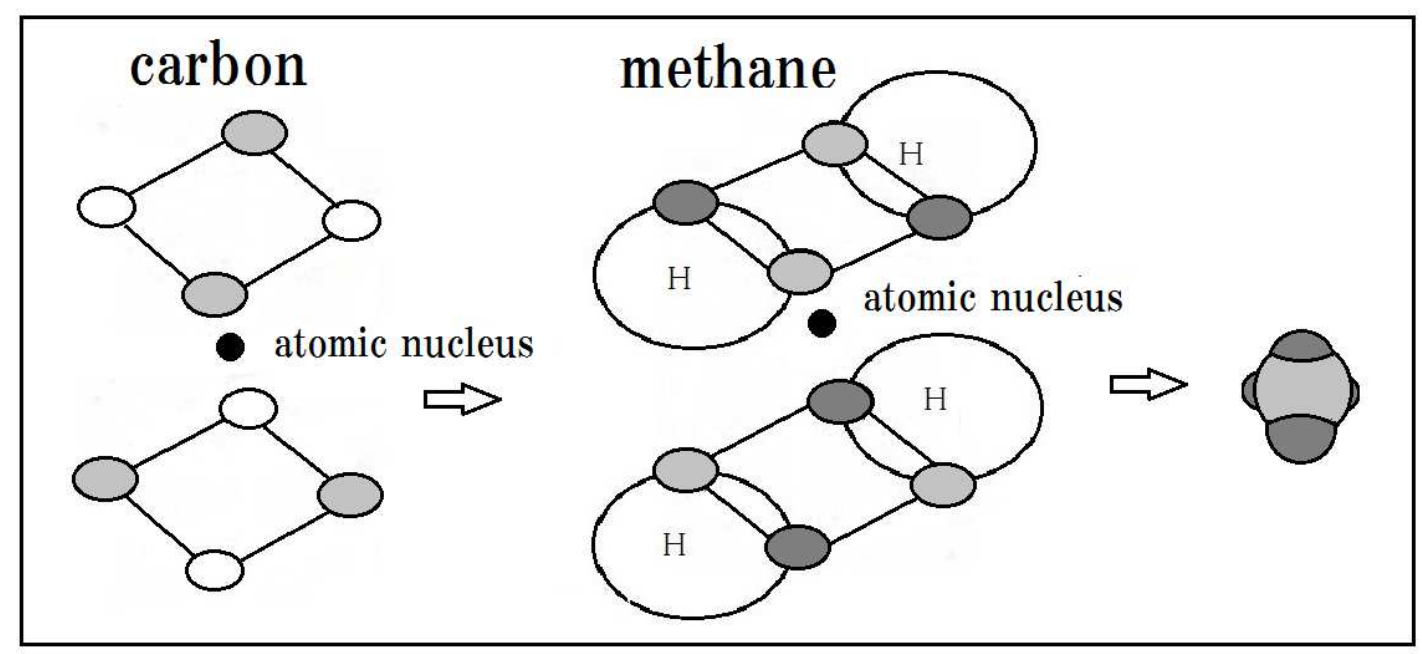

Fig. 3 methane

Figure 3 also suggests the three-dimensional structure of the methane molecule. That is, four hydrogen bonds are bonded at equal intervals around the carbon atom. That is, hydrogen is located at the apex of the regular tetrahedron, and carbon is present at the center.

The case of carbon monoxide (CO) will also be considered for further verification.

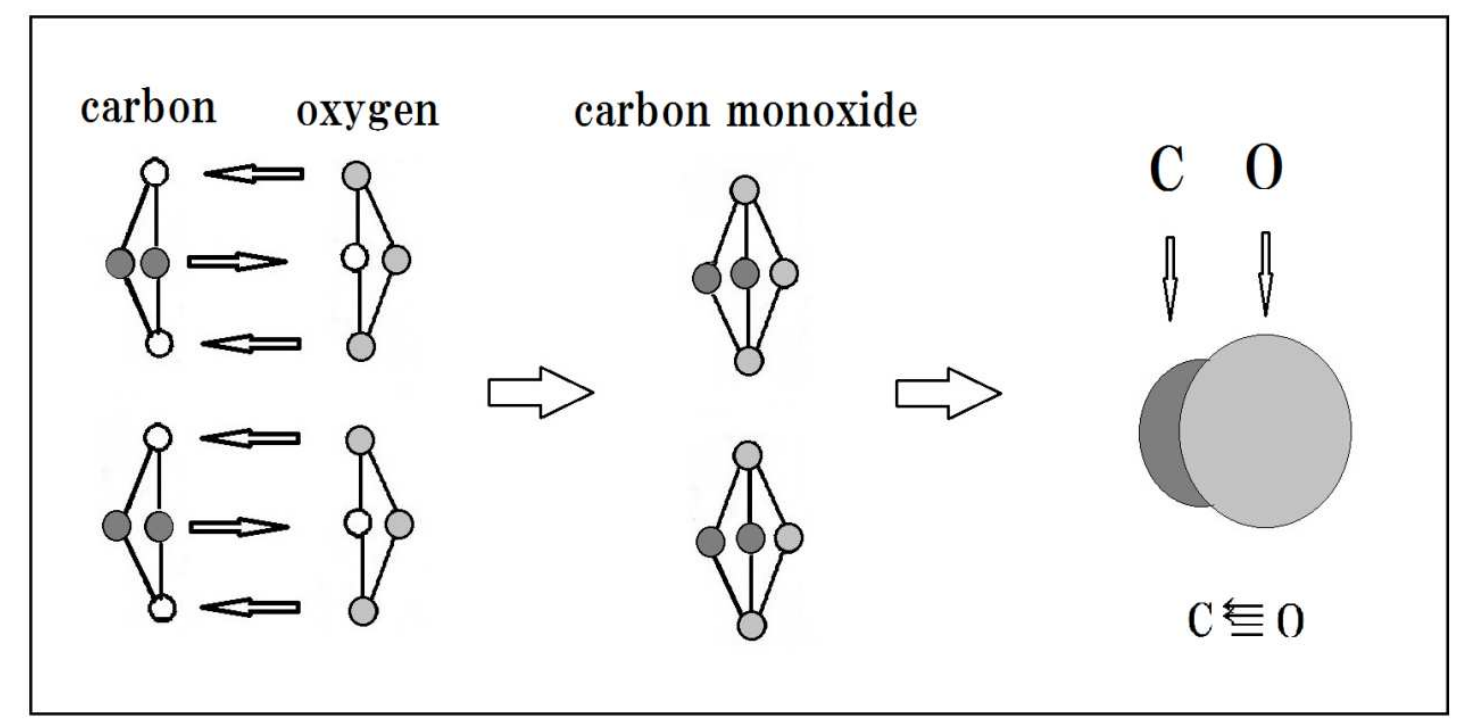

Fig. 4 carbon monoxide

The first small orbit passes two electrons from oxygen to carbon, and conversely one electron from carbon to oxygen, and the same phenomenon occurs in the second small orbit. As a result, all the electrons are buried in the small orbit, and it becomes an octet and stabilizes.

Therefore, carbon monoxide exists as a stable gas. However, it is not clear from the 
structural formula whether it is a stable gas.

The difference between the number of left-pointing arrows and the number of right-pointing arrows means that one element only donates an electron to the other element and does not receive it.

That is, in the case of carbon monoxide, there are two covalent bonds that exchange electrons, and the oxygen orbit is stable. On the other hand, the orbit of carbon is stable because oxygen gives carbon two electrons.

In the notation of this paper, it can be expressed as shown in Fig. 4.

Since carbon is tetravalent, it receives 4 electrons and is stable. That is, four lines are drawn, which means that tetravalent carbon receives four electrons from oxygen. This notation is very similar to the structural formula and is easy to understand graphically. On the other hand, oxygen shows an octet state by forming a double bond.

The arrow pointing to carbon means that it does not contribute to the stability of oxygen itself.

Using this notation, it is possible to express things that could not be expressed by the structural formula, so it can be said to be an extended Kekulé structural formula.

Figure 4 suggests the three-dimensional structure of carbon monoxide.

Next, hypochlorous acid ( $\mathrm{HClO})$ will be taken as an example in which the three-dimensional structure can be clearly understood.

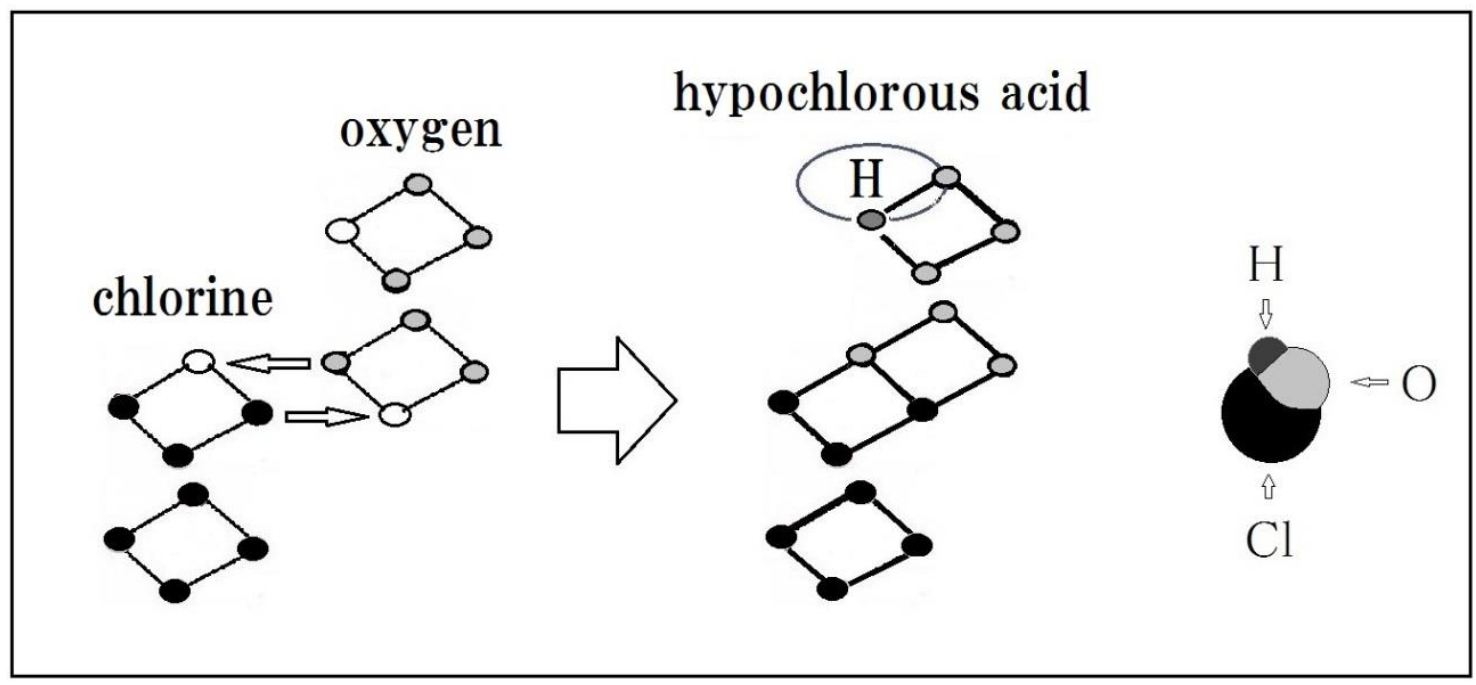

Fig. 5 hypochlorous acid

Chlorine has 7 valence electrons, oxygen has 6 valence electrons, and hydrogen has 1 valence electron.

One electron is passed from the second small orbit of oxygen to chlorine, and one electron is passed from the first small orbit of chlorine to oxygen. That is, oxygen to chlorine can be written as $\mathrm{O} \rightarrow \mathrm{Cl}$, and chlorine to oxygen can be written as $\mathrm{O} \leftarrow \mathrm{Cl}$. 
Chlorine and oxygen exchange electrons, so if you use the covalent bond symbol, it becomes $\mathrm{O}-\mathrm{Cl}$. Similarly, hydrogen and oxygen can be written as $\mathrm{H}-\mathrm{O}$, so hypochlorous acid becomes $\mathrm{H}-\mathrm{O}-\mathrm{Cl}$.

Figure 5 shows the three-dimensional structure of hypochlorous acid. That is, it can be seen that chlorine and hydrogen do not bond directly, and hydrogen, oxygen and chlorine are not on the same straight line.

Atoms with large valence electrons can hardly afford to receive from electrons to other atoms, but can donate as many electrons as there are valence electrons. The notation introduced in this paper expresses this number as the number of arrows $(\rightarrow)$. Therefore, the number can be used up to the number of valences.

Consider chlorous acid $\left(\mathrm{HClO}_{2}\right)$ as an example.

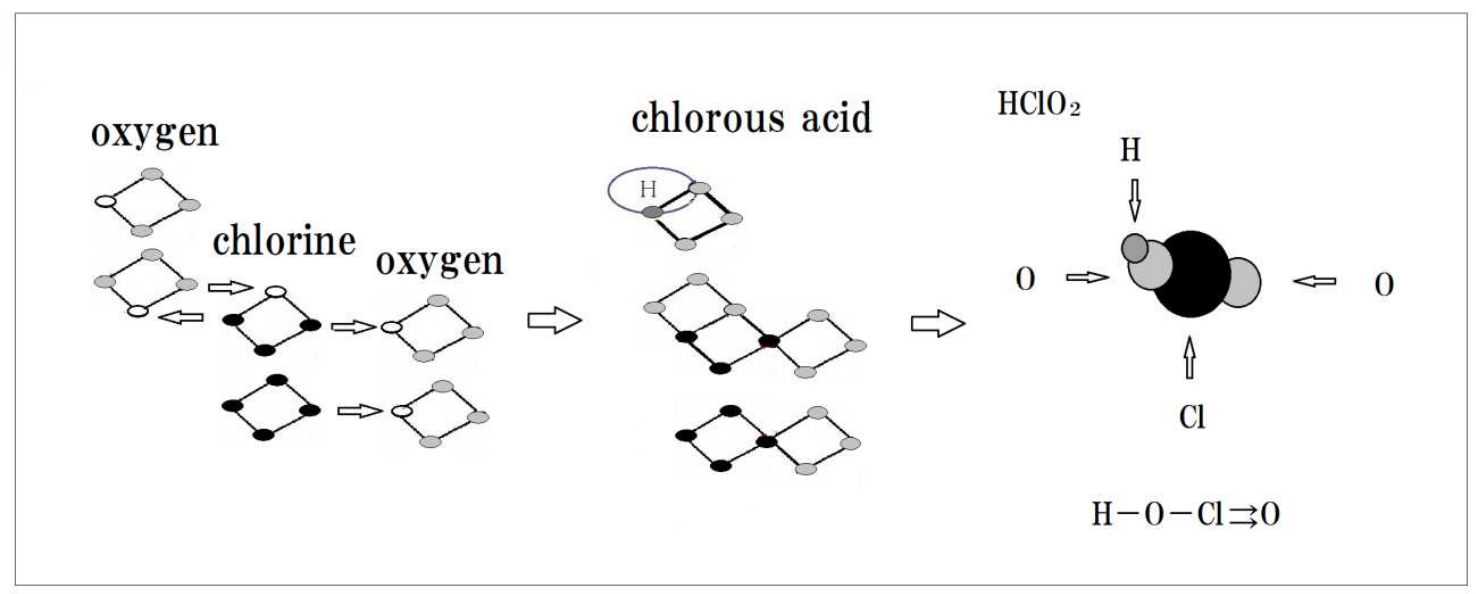

Fig. 6 chlorous acid

The structural formula of chlorous acid can be obtained by going through the same process as the example of hypochlorous acid.

The structural formula represents a three-dimensional figure.

One electron is passed from the first small orbit of the oxygen atom on the left side to the second small orbit of chlorine, and one electron is passed from the second small orbit of chlorine to oxygen.

Chlorine also transfers electrons to the first and second orbitals of oxygen on the right, so all orbitals are octets.

Expressed in the notation defined in this paper, the three-dimensional diagram of chlorous acid can be drawn as shown in Fig 6.

Figure 6 shows that the extended Kekule structural formula shows that the valence electrons of each element are bonded in just proportion, and that the three-dimensional diagram has a shape very close to the actual chlorous acid. 
(3) Valence electrons of transition elements

The periodic table was created by Mendelev focusing on the regularity of the valence electrons of the elements. However, transition elements have been treated as exceptional, even though there are many elements that do not have a fixed valence electron. Too many transition elements are used as discussed as exceptions.

Although the transition elements do not have the periodicity of valence electrons, Miura still felt a contradiction in what is called the periodic table, not the periodicity of the number of valence electrons, but the periodicity of the phenomenon called octet.

Miura's extended periodic table shows that neither the outermost electron nor the immediately adjacent inner shell electron is in the octet state when looking at the electron configuration of the transition element.

Conversely, all orbits of the inner shell of elements other than transition elements are in an octet state and are stable. Therefore, the electrons in the outermost shell cannot move to the inner shell. The electrons of elements belonging to transition elements can move to orbits that are not yet octets. That is, the valence electrons increase as the electrons in the inner shell move to the outermost shell. The reverse is also true.

The case of copper $(\mathrm{Cu})$ having an atomic number of 29 will be specifically shown.

The valence electrons of copper in the extended periodic table are divalent, with $\mathrm{N}_{X}$ orbitals being 2 and $\mathrm{L}_{\mathrm{Y}}$ orbitals being 7 .

Both orbitals are not octets, but since the energy order does not change much, the electrons in the $\mathrm{L}_{Y}$ orbit may move to the $\mathrm{N}_{X}$ orbit. If one electron is transferred, the valence electron of copper becomes trivalent, and if two electrons are transferred, the valence electron of copper becomes tetravalent.

By incorporating the phenomenon of octet stability in the extended periodic table, it is possible not only to reasonably explain the phenomenon of changes in the valence electrons of transition elements, but also to prove the periodicity of all elements.

(4) Periodic table of undiscovered elements (See Table 2)

In the current periodic table, the electron configuration of atoms up to the 118th element Oganesson $(\mathrm{Og})$ is announced. In addition, the electron configuration of undiscovered elements created according to the law is also created.

In this paper as well, the electron configuration of undiscovered elements was created according to Miura's hypothetical law.

As the number of electrons increases, the place where it is stored is stored in the outermost group 1 and 2 and then stored in the shell orbit inside it, and when it reaches the octet, it is stored in the next shell orbit. 
However, it was created according to the rule that only one orbit can enter the same shell.

As a result, the electron configuration of the outermost shell was almost the same up to the 136th element as compared with the conventional periodic table. However, the results were different for the elements after that.

This paper shows that the 142nd element is Group 18. This is a stable element with an octet outermost shell. However, in the conventional electron configuration, the outermost valence electron is divalent.

If the 142nd element is discovered in the future, the valence electrons of the outermost shell can be accurately obtained, and if it is a stable element, the results of this paper and Miura's hypothesis will be proved. 
Table 1 Extended Periodic Table

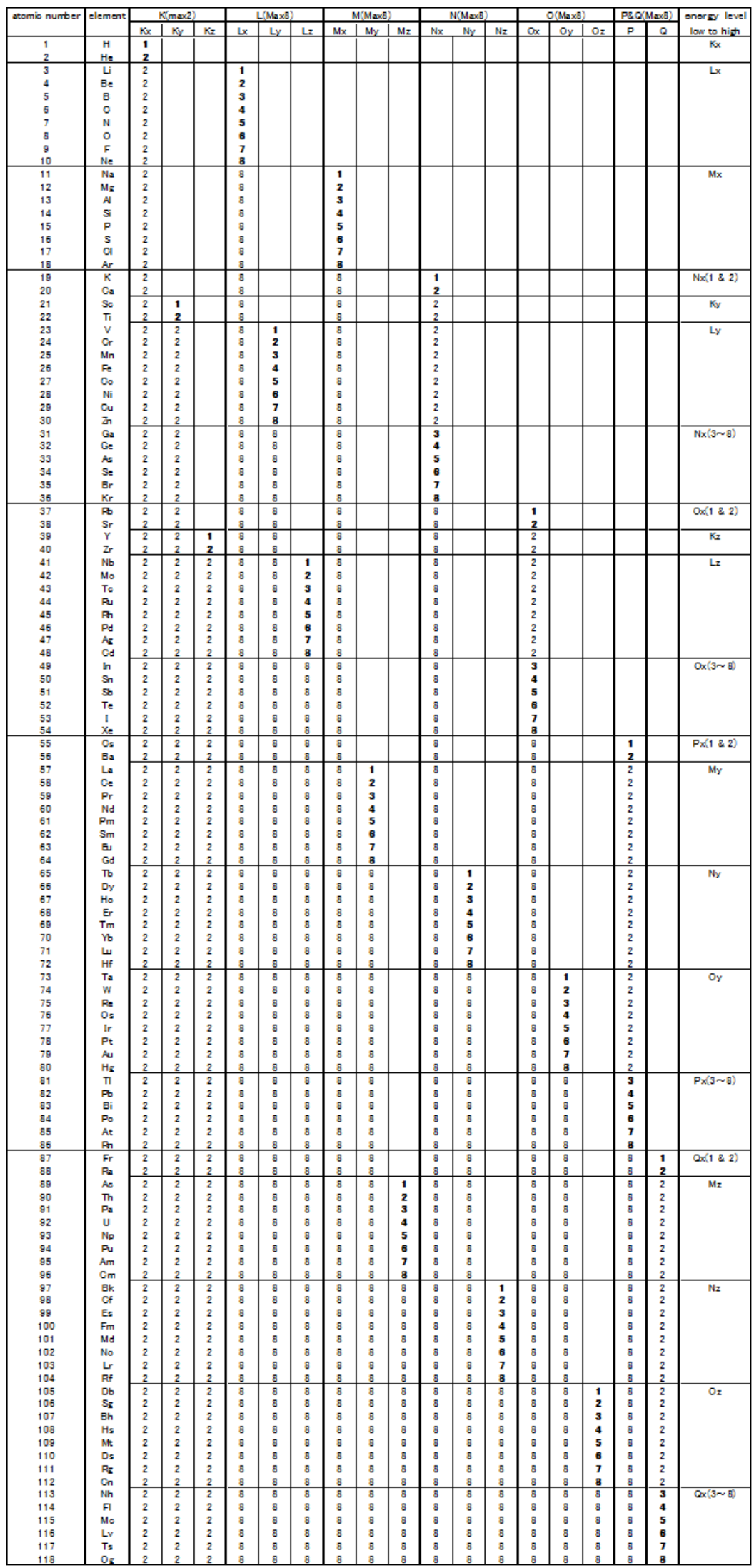




\section{Conclusion}

In this paper, we derived a new periodic table based on Miura's hypothesis (3 orbitals exist in each shell, and a barrier is formed in the shell when the octet is reached).

In addition, this extended periodic table clarified the energy level for the electrons in each shell to exist in orbit.

That is, the energy level is $\mathrm{K}_{\mathrm{X}}<\mathrm{L}_{\mathrm{X}}<\mathrm{M}_{\mathrm{X}}<\mathrm{N}_{\mathrm{X}}(1 \& 2)<\mathrm{K}_{\mathrm{Y}}<\mathrm{L}_{\mathrm{Y}}<\mathrm{N}_{\mathrm{X}}(3 \sim 8)<\mathrm{O}_{\mathrm{X}}(1 \& 2)<$ $\mathrm{K}_{\mathrm{Z}}<\mathrm{L}_{\mathrm{Z}}<\mathrm{O}_{\mathrm{X}}(3 \sim 8)<\mathrm{P}_{\mathrm{X}}(1 \& 2)<\mathrm{M}_{\mathrm{Y}}<\mathrm{N}_{\mathrm{Y}}<\mathrm{O}_{\mathrm{Y}}<\mathrm{P}_{\mathrm{X}}(3 \sim 8)<\mathrm{Q}_{\mathrm{X}}(1 \& 2)<\mathrm{M}_{\mathrm{Z}}<\mathrm{N}_{\mathrm{Z}}<\mathrm{O}_{\mathrm{Z}}<$ $\mathrm{Q}_{\mathrm{X}}(3 \sim 8)$.

Since this paper is a very simplified model, it seems that it is not so difficult to calculate the energy level.

Information such as the specifications required to obtain the energy level, that is, the distance from the electron and nucleus to each shell (radius to $\mathrm{K}, \mathrm{L}, \mathrm{M}, \mathrm{N}$, etc.) is already known. Therefore, it seems that the calculation will not be so complicated.

What is needed in this paper is to find the energy of the barrier formed in the shell when the orbit is filled with electrons and reaches the octet.

However, at this point, it seems that it will still take a considerable amount of time to create computer power and mathematical models. Therefore, by showing the usefulness of the extended periodic table created on the premise of Miura's hypothesis, we discussed the credibility without waiting for the calculation result.

Therefore, based on Miura's hypothesis, we added some restrictions and verified it.

We proposed a new notation to make its usefulness easier to understand.

When elements are represented using this notation, it is possible to visualize how electrons are arranged on two small orbitals centered on the atomic nucleus.

In addition, the process of bonding atoms to form molecules is easy to understand visually and serves as a three-dimensional diagram.

By expressing the molecule with the extended Kekule structural formula, it is possible to easily determine whether or not the molecule is in the octet state.

As a result of the above, for all the elements currently discovered, the extended periodic table based on Miura's hypothesis and the conventional periodic table are in excellent agreement.

In addition, the structural diagrams and structural formulas shown in this paper are consistent with the molecular structures constructed by introducing the concept of hybrid orbitals and the widely used structural formulas.

Therefore, Miura's hypothesis can clarify the definition of transition elements, and can easily predict the electron configuration of undiscovered elements. 
In Japan, groups 3 to 11 are classified as transition elements and group 12 is classified as a main group element, but there are also papers that classify group 12 as a transition element, and it is not unified worldwide.

It is extremely easy to understand that an element in which electrons that increase as the atomic number increases move to the inner shell instead of being stored in the outermost shell and are stored is defined as a transition element.

Therefore, it is considered that the elements of Group 12 should be classified as transition elements.

There are still many unknown areas in the current research in the atomic field, and we think that various researches are being carried out based on hypotheses.

It is extremely interesting to make a hypothesis about the electron configuration of an atom in this way, and to come across a new discovery, and to draw a consistent conclusion from that hypothesis. 
Table 2 Unknown element and electron configuration diagram

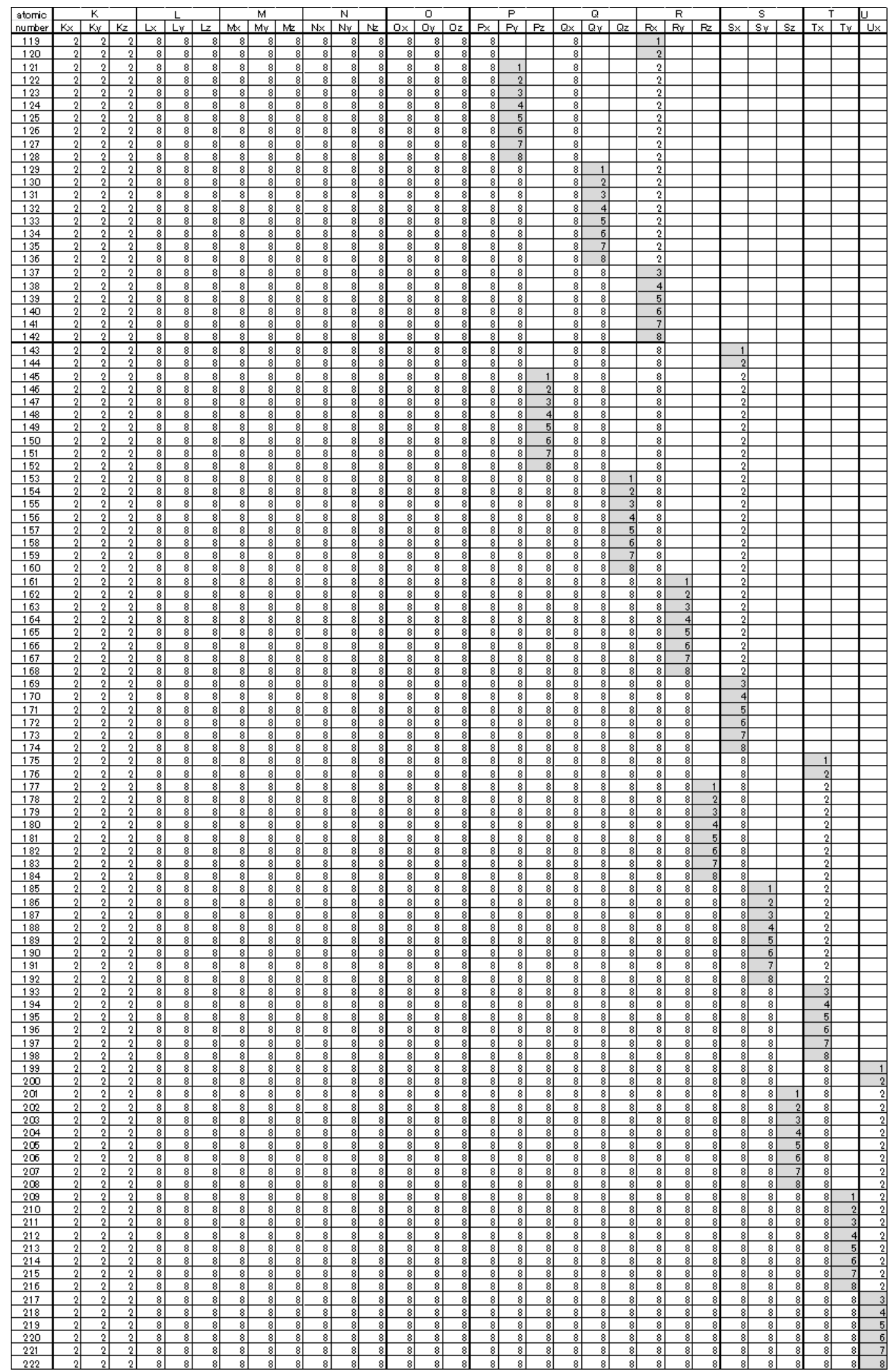

